\title{
Palladium-Catalyzed Cross-Coupling of $\alpha$-Diazocarbonyl Compounds with Arylboronic Acids
}

\author{
Cheng Peng, Yan Wang and Jianbo Wang* \\ Beijing National Laboratory of Molecular Sciences (BNLMS), Key Laboratory of Bioorganic Chemistry \\ and Molecular Engineering of Ministry of Education, College of Chemistry, Peking University, Beijing \\ 100871, China
}

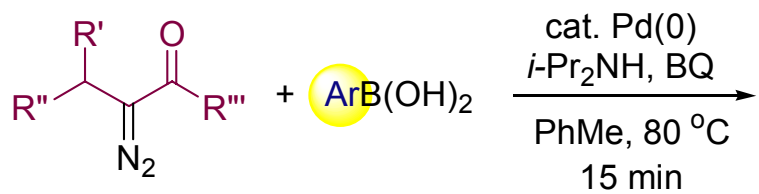<smiles>[R]C(=O)C([18O])=C([R])[R]</smiles>

$44-97 \%$

\section{CONTENTS}

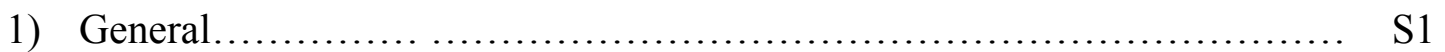

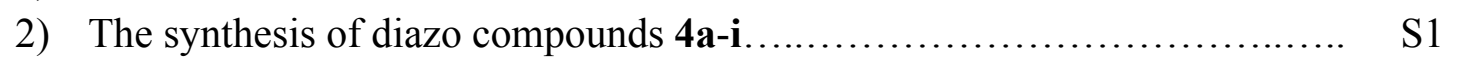

3) Spectral data of diazo compounds............................... S2

4) Typical procedure for Pd-catalyzed reaction.............................. S3

5) Spectral data for the cross-coupling products........................ S3

6) $\mathrm{Pd}\left(\mathrm{PPh}_{3}\right)_{4}$-catalyzed reaction of 1 with iodobenzene................... S7

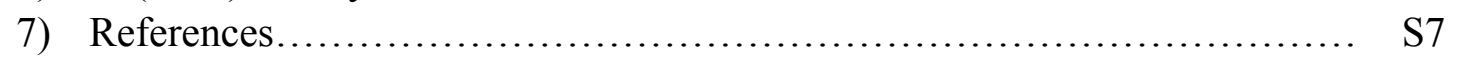


General All reactions were performed under a nitrogen atmosphere in a flame-dried reaction flask. All solvents were distilled prior to use. Toluene and THF were dried over Na with benzophenone-ketyl intermediate as indicator. For chromatography, 200-300 mesh silica gel (Qingdao, China) was employed. ${ }^{1} \mathrm{H}$ and ${ }^{13} \mathrm{C}$ NMR spectra were recorded at $300 \mathrm{MHz}$ (or 200 $\mathrm{MHz}$ ) and $75 \mathrm{MHz}$ (or $50 \mathrm{MHz}$ ) with Varian Mercury 300 spectrometer. Chemical shifts are reported in ppm using tetramethylsilane as internal standard. IR spectra were recorded with a Nicolet 5MX-S infrared spectrometer.

\section{The Synthesis of Diazo Compounds 4a, b, d, e, g, h.}

4a was prepared following the procedure reported by Moody and coworkers. ${ }^{1}$

The preparation of $\mathbf{4 b} \mathbf{b}, \mathbf{d}$, and $\mathbf{e}$ is followed by the scheme shown below.

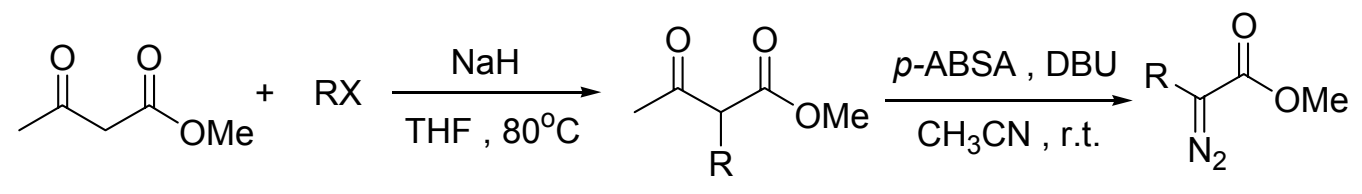

$$
\begin{aligned}
& \mathrm{RX}: \sim^{1} \mathbf{6 a} \quad 7 \mathrm{a} \text {, Colorless liquid, } 92 \% \text { 4b, } 51 \% \\
& \mathrm{Ph} \sim \mathrm{Br} \text { 6b } \quad 7 b \text {, Colorless liquid, } 71 \% \text { 4d, } 69 \% \\
& \begin{array}{lllll}
-1 & 6 c & 7 c & - & 4 e, 14 \%^{a}
\end{array}
\end{aligned}
$$

(a) Under a nitrogen atmosphere, a solution of methyl 3-oxobutanoate $(3.48 \mathrm{~g}, 30 \mathrm{mmol})$ in THF $(10 \mathrm{~mL})$ was added dropwise to a stirred suspension of $\mathrm{NaH}(60 \%$ dispersion in mineral oil, $1.2 \mathrm{~g}, 30 \mathrm{mmol})$ in THF $(10 \mathrm{~mL})$ at room temperature. After the mixture became clear, a solution of RX $(20 \mathrm{mmol})$ in THF $(10 \mathrm{~mL})$ was added dropwise, and the mixture was stirred at $80{ }^{\circ} \mathrm{C}$. After completion of the reaction as monitored by TLC, saturated aqueous $\mathrm{NH}_{4} \mathrm{Cl}$ solution $(10 \mathrm{~mL})$ was added, and the mixture was extracted with dichloromethane. The combined organic layers were dried over $\mathrm{Na}_{2} \mathrm{SO}_{4}$ and evaporated, and the residue was purified by column chromatography to afford the pure product $7 \mathbf{a}$ and $7 \mathbf{b}$.

(b) To a solution of 7a $\sim \mathbf{c}(10 \mathrm{mmol})$ and DBU $(2.28 \mathrm{~g}, 15 \mathrm{mmol})$ in $\mathrm{MeCN}(10 \mathrm{~mL})$, was added dropwise $p$-acetamidobenzenesulfonyl azide ( $p$-ABSA) $(3.12 \mathrm{~g}, 13 \mathrm{mmol})$ in $\mathrm{MeCN}$ $(10 \mathrm{~mL})$ at room temperature overnight. Crude purification by flash column chromatography removed DBU, and the purification by column chromatography gave the pure $\mathbf{4 b}, \mathbf{d}, \mathbf{e}$.

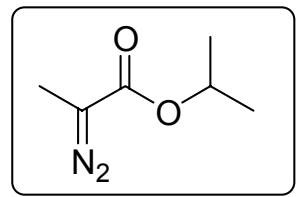

found 142.0745 .

\section{Isopropyl 2-diazopropionate (4a)}

Yellow liquid. ${ }^{1} \mathrm{H}$ NMR $\left(300 \mathrm{MHz}, \mathrm{CDCl}_{3}\right) \delta 1.25(\mathrm{~d}, J=6.3 \mathrm{~Hz}, 6 \mathrm{H})$, $1.95(\mathrm{~s}, 3 \mathrm{H}), 5.08(\mathrm{~m}, 1 \mathrm{H}) ;{ }^{13} \mathrm{C} \mathrm{NMR}\left(75 \mathrm{MHz}, \mathrm{CDCl}_{3}\right) \delta: 8.35,22.0,68.2$; IR (film): 2982, 2078, 1686, $1140 \mathrm{~cm}^{-1}$; EI-MS ( $\mathrm{m} / \mathrm{z}$, relative intensity): $142\left(\mathrm{M}^{+}, 27\right), 83$ (30), 43 (100). HRMS cacld for $\mathrm{C}_{6} \mathrm{H}_{10} \mathrm{~N}_{2} \mathrm{O}_{2}$ 142.0742, 


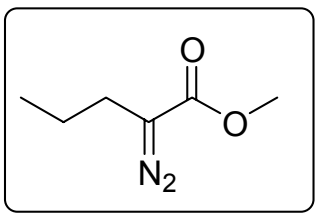

Methyl 2-diazopentanoate (4b)

Yellow liquid. ${ }^{1} \mathrm{H}$ NMR (300 $\left.\mathrm{MHz}, \mathrm{CDCl}_{3}\right) \delta 0.97(\mathrm{t}, J=7.5 \mathrm{~Hz}, 3 \mathrm{H})$, $1.48 \sim 1.61(\mathrm{~m}, 2 \mathrm{H}), 2.29(\mathrm{t}, J=7.5 \mathrm{~Hz}, 2 \mathrm{H}), 3.77(\mathrm{~s}, 3 \mathrm{H}) ;{ }^{13} \mathrm{C} \mathrm{NMR}(75$ $\left.\mathrm{MHz}, \mathrm{CDCl}_{3}\right) \delta 13.2,20.9,25.0,51.8,168.1$; IR (film): 2962, 2078, $1695,1136 \mathrm{~cm}^{-1}$; EI-MS ( $\mathrm{m} / \mathrm{z}$, relative intensity): $142\left(\mathrm{M}^{+}, 48\right), 83(47)$, 55 (100). Anal. cacld for $\mathrm{C}_{6} \mathrm{H}_{10} \mathrm{~N}_{2} \mathrm{O}_{2}$ : C, 50.69; H, 7.09; N, 19.71. Found C, 50.62; H, 6.99; N, 19.79 .

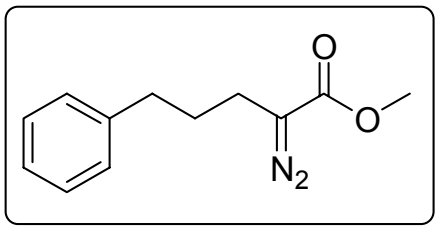

\section{Methyl 2-diazo-5-phenylpentanoate (4d)}

Yellow liquid. ${ }^{1} \mathrm{H}$ NMR $\left(300 \mathrm{MHz}, \mathrm{CDCl}_{3}\right) \delta 1.84(\mathrm{~m}, 2 \mathrm{H}), 2.34$ (t, $J=7.5 \mathrm{~Hz}, 2 \mathrm{H}), 2.67(\mathrm{t}, J=7.5 \mathrm{~Hz}, 2 \mathrm{H}), 3.75(\mathrm{~s}, 3 \mathrm{H})$, 7.17 7.31 (m, 5H); ${ }^{13} \mathrm{C}$ NMR $\left(75 \mathrm{MHz}, \mathrm{CDCl}_{3}\right) \delta 22.7,29.1$, 34.8, 51.8, 125.9, 128.2, 128.3, 141.2, 167.8; IR (film): 2950, 2078, 1691, $1114 \mathrm{~cm}^{-1}$; EI-MS ( $\mathrm{m} / z$, relative intensity): 190 [(M-28) $\left.{ }^{+}, 17\right], 158(35), 131(85)$, 91 (100). Anal. calcd for $\mathrm{C}_{12} \mathrm{H}_{14} \mathrm{~N}_{2} \mathrm{O}_{2}$ : C, 66.04; H, 6.47; N, 12.84. Found C, 66.01; H, 6.40; $\mathrm{N}, 12.92$.

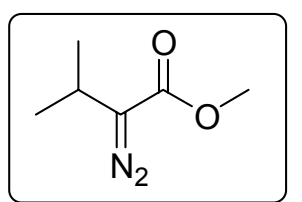

\section{Methyl 2-diazo-3-methylbutanoate (4e)}

Yellow liquid. ${ }^{1} \mathrm{H}$ NMR $\left(300 \mathrm{MHz}, \mathrm{CDCl}_{3}\right) \delta 1.15(\mathrm{~d}, J=6.9 \mathrm{~Hz}, 6 \mathrm{H})$, $2.76(\mathrm{~m}, 1 \mathrm{H}), 3.77(\mathrm{~s}, 3 \mathrm{H}) ;{ }^{13} \mathrm{C} \mathrm{NMR}\left(75 \mathrm{MHz}, \mathrm{CDCl}_{3}\right) \delta 20.4,23.1,51.6$, 167.4; IR (film): 2959, 2077, 1693, $1270 \mathrm{~cm}^{-1}$; EI-MS $(\mathrm{m} / z$, relative intensity): $142\left(\mathrm{M}^{+}, 14\right), 111$ (21), 99 (24), 83 (100), 55 (32). HRMS calcd for $\mathrm{C}_{6} \mathrm{H}_{10} \mathrm{~N}_{2} \mathrm{O}_{2}$ 142.0742, found 142.0742.

$\mathbf{4 g}$ and $\mathbf{4 h}$ were prepared following the procedure reported by Taber and coworkers. ${ }^{2}$

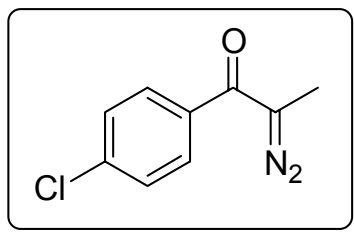

\section{1-(4-Chlorophenyl)-2-diazo-propan-1-one (4g)}

Yellow solid. ${ }^{1} \mathrm{H}$ NMR $\left(300 \mathrm{MHz}, \mathrm{CDCl}_{3}\right) \delta 2.14(\mathrm{~s}, 3 \mathrm{H}), 7.41(\mathrm{~d}, J=$ $8.4 \mathrm{~Hz}, 2 \mathrm{H}), 7.54(\mathrm{~d}, J=8.4 \mathrm{~Hz}, 2 \mathrm{H}) ;{ }^{13} \mathrm{C} \mathrm{NMR}\left(75 \mathrm{MHz}, \mathrm{CDCl}_{3}\right) \delta$ 9.4, 62.9, 128.5, 128.7, 135.7, 137.3, 188.5; IR (film): 2930, 2070, 1604, $1339 \mathrm{~cm}^{-1}$; EI-MS ( $\mathrm{m} / \mathrm{z}$, relative intensity): $194\left(\mathrm{M}^{+}, 9\right), 166$ (21), 138 (87), 111 (33), 103 (100), 75 (49); HRMS calcd for $\mathrm{C}_{9} \mathrm{H}_{7} \mathrm{~N}_{2} \mathrm{OCl}$ 194.0247, found 194.0248 .

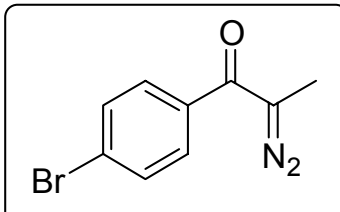

\section{1-(4-Bromophenyl) -2-diazo-propan-1-one (4h)}

Yellow solid. ${ }^{1} \mathrm{H}$ NMR $\left(300 \mathrm{MHz}, \mathrm{CDCl}_{3}\right) \delta 2.14(\mathrm{~s}, 3 \mathrm{H}), 7.46(\mathrm{~d}, J=$ $8.1 \mathrm{~Hz}, 2 \mathrm{H}), 7.58(\mathrm{~d}, J=8.1 \mathrm{~Hz}, 2 \mathrm{H}) ;{ }^{13} \mathrm{C} \mathrm{NMR}\left(75 \mathrm{MHz}, \mathrm{CDCl}_{3}\right) \delta$ 9.5, 63.0, 125.8, 128.7, 131.7, 136.2, 188.7; IR (film): 2922, 2069, $1605,1338 \mathrm{~cm}^{-1}$; EI-MS ( $\mathrm{m} / \mathrm{z}$, relative intensity): $238\left(\mathrm{M}^{+}, 5\right), 210$ (10), 182 (49), 155 (14), 103 (100), 77 (55); HRMS calcd for $\mathrm{C}_{9} \mathrm{H}_{7} \mathrm{~N}_{2} \mathrm{OBr}$ 237.9742, found 237.9748 . 


\section{Typical Procedure for the Pd-Catalyzed Cross-Coupling Reaction.}

Under a nitrogen atmosphere, to a mixture of phenylboronic acid (2a, $183 \mathrm{mg}, 1.5 \mathrm{mmol})$, $i$ - $\mathrm{Pr}_{2} \mathrm{NH}$ (253 mg, $\left.2.5 \mathrm{mmol}\right)$, BQ (81 mg, $\left.0.75 \mathrm{mmol}\right), \mathrm{Pd}\left(\mathrm{PPh}_{3}\right)_{4}(14 \mathrm{mg}, 0.0125 \mathrm{mmol})$ in $\mathrm{PhMe}(10 \mathrm{~mL})$ was added methyl $\alpha$-diazopropionate $(1,57 \mathrm{mg}, 0.5 \mathrm{mmol})$. The mixture was stirred at $80^{\circ} \mathrm{C}$ until 1 disappeared as judged by TLC (15 min). The solvent was evaporated in vacuo. Purification by column chromatography of the mixture gave the pure 3a as a colorless oil (66 mg, $82 \%)$.

Use of smaller amount of phenylboronic acid 2a (2.0 and 1.1 eq.) under the otherwise identical conditions resulted in diminished yields of 3a (76\% and $52 \%$, respectively), and longer reaction time.

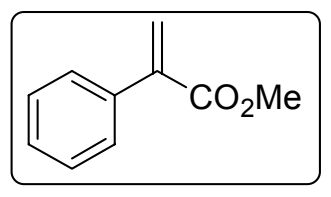

Methyl 2-phenylacrylate (3a) ${ }^{3}$

Colorless oil. ${ }^{1} \mathrm{H}$ NMR (300 MHz, $\left.\mathrm{CDCl}_{3}\right) \delta 3.82(\mathrm{~s}, 3 \mathrm{H}), 5.89(\mathrm{~d}, J=$ $0.9 \mathrm{~Hz}, 1 \mathrm{H}), 6.36(\mathrm{~d}, J=0.9 \mathrm{~Hz}, 1 \mathrm{H}), 7.33 \sim 7.43(\mathrm{~m}, 5 \mathrm{H}) ;{ }^{13} \mathrm{C}$ NMR $(75$ $\left.\mathrm{MHz}, \mathrm{CDCl}_{3}\right) \delta 52.2,126.9,128.1,128.2,128.3,136.7,141.3,167.2$; IR (film): 2952, 1721, $1201 \mathrm{~cm}^{-1}$; EI-MS ( $\mathrm{m} / \mathrm{z}$, relative intensity): $162\left(\mathrm{M}^{+}, 49\right), 131$ (13), 103 (100), 77 (30).

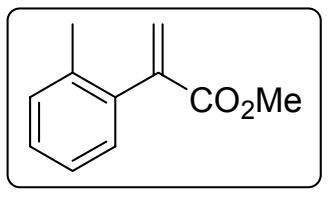

Methyl 2-o-tolylacrylate (3b)

Colorless oil. ${ }^{1} \mathrm{H}$ NMR $\left(300 \mathrm{MHz}, \mathrm{CDCl}_{3}\right) \delta 2.19$ (s, 3H), 3.75 (s, 3H), $5.70(\mathrm{~d}, J=1.5 \mathrm{~Hz}, 1 \mathrm{H}), 6.51(\mathrm{~d}, J=1.5 \mathrm{~Hz}, 1 \mathrm{H}), 7.11 \sim 7.27(\mathrm{~m}, 4 \mathrm{H})$; ${ }^{13} \mathrm{C}$ NMR $\left(75 \mathrm{MHz}, \mathrm{CDCl}_{3}\right) \delta 19.7,52.1,125.6,128.1,128.6,129.4$, 129.8, 136.0, 137.1, 141.7, 167.1; IR (film): 2951, 1722, $1209 \mathrm{~cm}^{-1}$; EI-MS ( $\mathrm{m} / z$, relative intensity): 176 (M+, 72$), 161$ (19), 144 (14), 117 (78), 115 (100), 91 (27).

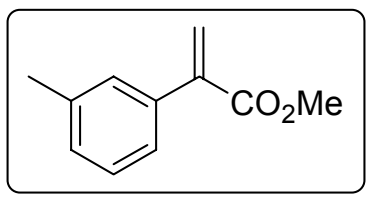

\section{Methyl 2-m-tolylacrylate (3c) ${ }^{5}$}

Pale yellow oil. ${ }^{1} \mathrm{H}$ NMR $\left(300 \mathrm{MHz}, \mathrm{CDCl}_{3}\right) \delta 2.37(\mathrm{~s}, 3 \mathrm{H}), 3.82(\mathrm{~s}$, $3 \mathrm{H}), 5.87(\mathrm{~d}, J=0.9 \mathrm{~Hz}, 1 \mathrm{H}), 6.34(\mathrm{~d}, J=0.9 \mathrm{~Hz}, 1 \mathrm{H}), 7.15 \sim 7.26(\mathrm{~m}$, $4 \mathrm{H}) ;{ }^{13} \mathrm{C}$ NMR $\left(75 \mathrm{MHz}, \mathrm{CDCl}_{3}\right) \delta 21.4,52.1,125.3,126.6,128.0$, 128.9, 129.0, 136.6, 137.7, 141.4, 167.4; IR (film): 2951, 1724, 1225, $1162 \mathrm{~cm}^{-1}$; EI-MS ( $\mathrm{m} / z$, relative intensity): $176\left(\mathrm{M}^{+}, 70\right), 145(8), 117(100), 91$ (16).

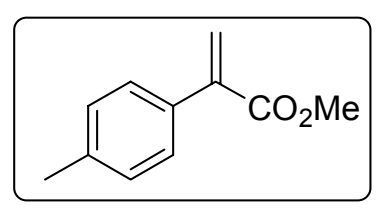

\section{Methyl 2-p-tolylacrylate (3d) ${ }^{6}$}

Pale yellow oil. ${ }^{1} \mathrm{H}$ NMR $\left(300 \mathrm{MHz}, \mathrm{CDCl}_{3}\right) \delta 2.36(\mathrm{~s}, 3 \mathrm{H}), 3.81(\mathrm{~s}$, $3 \mathrm{H}), 5.85(\mathrm{~d}, J=1.2 \mathrm{~Hz}, 1 \mathrm{H}), 6.31(\mathrm{~d}, J=1.2 \mathrm{~Hz}, 1 \mathrm{H}), 7.16(\mathrm{~d}, J=$ $7.8 \mathrm{~Hz}, 2 \mathrm{H}), 7.30(\mathrm{~d}, J=7.8 \mathrm{~Hz}, 2 \mathrm{H}) ;{ }^{13} \mathrm{C} \mathrm{NMR}\left(75 \mathrm{MHz}, \mathrm{CDCl}_{3}\right) \delta$ 21.1, 52.1, 126.1, 128.1, 128.8, 133.8, 138.0, 141.1, 167.4; IR (film): 2951, 1723, 1202, $1089 \mathrm{~cm}^{-1}$; EI-MS ( $\mathrm{m} / z$, relative intensity): $176\left(\mathrm{M}^{+}, 63\right), 117(100), 91$ (16).

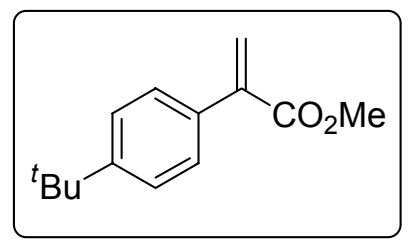

\section{Methyl 2-(4-tert-butylphenyl)acrylate (3e)}

Colorless oil. ${ }^{1} \mathrm{H}$ NMR (300 MHz, $\left.\mathrm{CDCl}_{3}\right) \delta 1.33$ (s, 9H), 3.82 (s, $3 \mathrm{H}), 5.88(\mathrm{~d}, J=1.2 \mathrm{~Hz}, 1 \mathrm{H}), 6.32(\mathrm{~d}, J=1.2 \mathrm{~Hz}, 1 \mathrm{H}), 7.34 \sim 7.41$ $(\mathrm{m}, 4 \mathrm{H}) ;{ }^{13} \mathrm{C} \mathrm{NMR}\left(75 \mathrm{MHz}, \mathrm{CDCl}_{3}\right) \delta 31.2,34.5,52.1,125.1$, 
126.2, 127.9, 133.7, 141.0, 151.2, 167.4; IR (film): 2961, 1724, 1208, $1115 \mathrm{~cm}^{-1}$; EI-MS $(\mathrm{m} / z$, relative intensity): $218\left(\mathrm{M}^{+}, 20\right), 203$ (100), 159 (5), 143 (24); HRMS calcd for $\mathrm{C}_{14} \mathrm{H}_{18} \mathrm{O}_{2}$ 218.1307, found 218.1309.

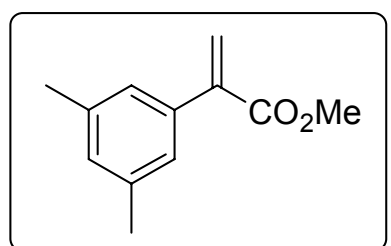

(100), 91 (17); HRMS calcd for $\mathrm{C}_{12} \mathrm{H}_{14} \mathrm{O}_{2}$ 190.0994, found 190.0994.

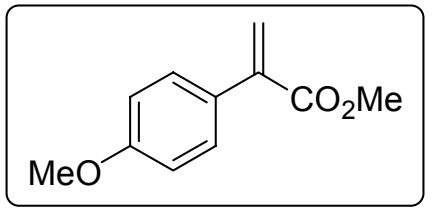

\section{Methyl 2-(4-methoxyphenyl)acrylate (3g) ${ }^{7}$}

Colorless oil. ${ }^{1} \mathrm{H}$ NMR $\left(300 \mathrm{MHz}, \mathrm{CDCl}_{3}\right) \delta 3.81(\mathrm{~s}, 3 \mathrm{H}), 3.82$ (s, $3 \mathrm{H}), 5.83(\mathrm{~d}, J=1.2 \mathrm{~Hz}, 1 \mathrm{H}), 6.26(\mathrm{~d}, J=1.2 \mathrm{~Hz}, 1 \mathrm{H}), 6.88(\mathrm{~d}, J$ $=9.0 \mathrm{~Hz}, 2 \mathrm{H}), 7.36(\mathrm{~d}, J=9.0 \mathrm{~Hz}, 2 \mathrm{H}) ;{ }^{13} \mathrm{C} \mathrm{NMR}(75 \mathrm{MHz}$, $\left.\mathrm{CDCl}_{3}\right) \delta 52.1,55.2,113.5,125.3,129.1,129.4,140.6,159.6$, 167.5; IR (film): 2953, 1721, 1512, $1173 \mathrm{~cm}^{-1}$; EI-MS ( $\mathrm{m} / z$, relative intensity): $192\left(\mathrm{M}^{+}, 68\right)$, 133 (100), $118(9), 92(5)$.

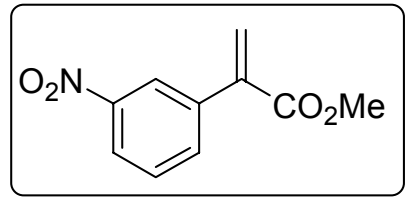

\section{Methyl 2-(3-nitrophenyl)acrylate (3h)}

White solid. ${ }^{1} \mathrm{H}$ NMR (300 MHz, $\left.\mathrm{CDCl}_{3}\right) \delta 3.87$ (s, 3H), 6.05 (s, $1 \mathrm{H}), 6.55(\mathrm{~s}, 1 \mathrm{H}), 7.53 \sim 7.58(\mathrm{~m}, 1 \mathrm{H}), 7.76 \sim 7.80(\mathrm{~m}, 1 \mathrm{H})$, $8.19 \sim 8.23(\mathrm{~m}, 1 \mathrm{H}), 8.31 \sim 8.32(\mathrm{~m}, 1 \mathrm{H}) ;{ }^{13} \mathrm{C} \mathrm{NMR}\left(75 \mathrm{MHz}, \mathrm{CDCl}_{3}\right)$ $\delta$ 52.4, 122.9, 123.3, 129.0, 129.2, 134.4, 138.2, 139.1, 148.0, 166.0; IR (film): 2954, 1724, 1529, 1350, $1207 \mathrm{~cm}^{-1}$; EI-MS ( $\mathrm{m} / z$, relative intensity): $207\left(\mathrm{M}^{+}, 100\right), 176(8), 148(35), 102$ (20); HRMS calcd for $\mathrm{C}_{10} \mathrm{H}_{9} \mathrm{NO}_{4} 207.0532$, found 207.0527.

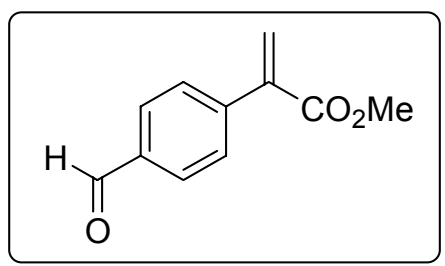

\section{Methyl 2-(4-formylphenyl)acrylate (3i)}

White solid. ${ }^{1} \mathrm{H}$ NMR (300 MHz, $\left.\mathrm{CDCl}_{3}\right) \delta 3.85$ (s, 3H), 6.02 (s, $1 \mathrm{H}), 6.51(\mathrm{~s}, 1 \mathrm{H}), 7.60(\mathrm{~d}, J=8.1 \mathrm{~Hz}, 2 \mathrm{H}), 7.89$ (d, $J=8.1 \mathrm{~Hz}$, 2H), $10.04(\mathrm{~s}, 1 \mathrm{H}) ;{ }^{13} \mathrm{C} \mathrm{NMR}\left(75 \mathrm{MHz}, \mathrm{CDCl}_{3}\right) \delta 52.3,128.8$, 129.0, 129.4, 135.8, 140.2, 142.6, 166.3, 191.7; IR (film): 2954, $1709,1687,1204,1090 \mathrm{~cm}^{-1}$; EI-MS ( $\mathrm{m} / z$, relative intensity): $190\left(\mathrm{M}^{+}, 100\right), 159$ (13), 131 (72), 103 (24); HRMS calcd for $\mathrm{C}_{11} \mathrm{H}_{10} \mathrm{O}_{3}$ 190.0630, found 190.0635 .

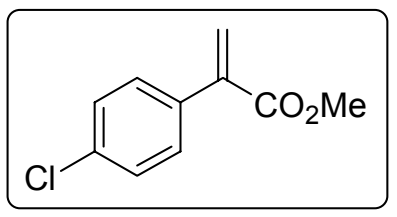

\section{Methyl 2-(4-chlorophenyl)acrylate (3j) $)^{8}$}

Colorless oil. ${ }^{1} \mathrm{H}$ NMR $\left(300 \mathrm{MHz}, \mathrm{CDCl}_{3}\right) \delta 3.83$ (s, 3H), 5.90 (d, $J$ $=0.9 \mathrm{~Hz}, 1 \mathrm{H}), 6.39(\mathrm{~d}, J=0.9 \mathrm{~Hz}, 1 \mathrm{H}), 7.33 \sim 7.36(\mathrm{~m}, 4 \mathrm{H}) ;{ }^{13} \mathrm{C}$ NMR $\left(75 \mathrm{MHz}, \mathrm{CDCl}_{3}\right) \delta 52.2,127.3,128.3,129.6,134.2,135.1$, 140.1, 166.8; IR (film): 2952, 1722, 1202, $1094 \mathrm{~cm}^{-1}$; EI-MS $(\mathrm{m} / z$, relative intensity): $196\left(\mathrm{M}^{+}, 67\right), 137$ (100), 111 (14). 


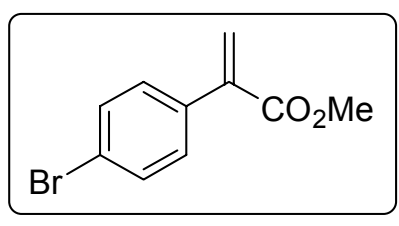

Methyl 2-(4-bromophenyl)acrylate (3k)

Colorless oil. ${ }^{1} \mathrm{H}$ NMR $\left(300 \mathrm{MHz}, \mathrm{CDCl}_{3}\right) \delta 3.82(\mathrm{~s}, 3 \mathrm{H}), 5.90(\mathrm{~d}, J$ $=0.9 \mathrm{~Hz}, 1 \mathrm{H}), 6.39(\mathrm{~d}, J=0.9 \mathrm{~Hz}, 1 \mathrm{H}), 7.27 \sim 7.31(\mathrm{~m}, 2 \mathrm{H})$, 7.47 7.49 (m, 2H); ${ }^{13} \mathrm{C}$ NMR (75 MHz, $\left.\mathrm{CDCl}_{3}\right) \delta 52.3,122.4$, 127.4, 129.9, 131.2, 135.5, 140.2, 166.7; IR (film): 2951, 1722, 1202, $1090 \mathrm{~cm}^{-1}$; EI-MS (m/z, relative intensity): 240 ( $\left.\mathrm{M}^{+}, 97\right), 181$ (94), 102 (100), 75 (29).

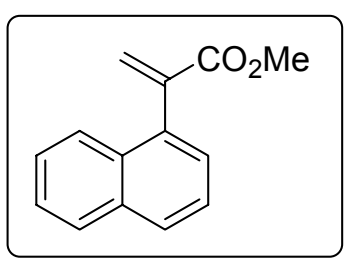

\section{Methyl 2-(naphthalen-1-yl)acrylate (31) ${ }^{9}$}

Colorless oil. ${ }^{1} \mathrm{H}$ NMR $\left(300 \mathrm{MHz}, \mathrm{CDCl}_{3}\right) \delta 3.71(\mathrm{~s}, 3 \mathrm{H}), 5.88(\mathrm{~d}, J=$ $1.8 \mathrm{~Hz}, 1 \mathrm{H}), 6.72(\mathrm{~d}, J=1.8 \mathrm{~Hz}, 1 \mathrm{H}), 7.34 \sim 7.37(\mathrm{~m}, 1 \mathrm{H}), 7.43 \sim 7.50$ $(\mathrm{m}, 3 \mathrm{H}), 7.71 \sim 7.75(\mathrm{~m}, 1 \mathrm{H}), 7.83 \sim 7.87(\mathrm{~m}, 2 \mathrm{H}) ;{ }^{13} \mathrm{C} \mathrm{NMR}(75 \mathrm{MHz}$, $\left.\mathrm{CDCl}_{3}\right) \delta 52.2,125.2,125.8,126.1,126.9,128.3,128.5,129.9,131.7$, 133.3, 135.2, 140.6, 167.4; IR (film): 2950, 1721, 1222, $\mathrm{cm}^{-1}$; EI-MS ( $\mathrm{m} / \mathrm{z}$, relative intensity): $212\left(\mathrm{M}^{+}, 28\right), 153(100), 84(27)$.

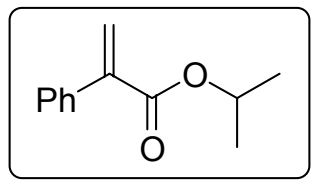

\section{Isopropyl 2-phenylacrylate (5a)}

Colorless oil. ${ }^{1} \mathrm{H}$ NMR (300 MHz, $\left.\mathrm{CDCl}_{3}\right) \delta 1.31(\mathrm{~d}, J=6.3 \mathrm{~Hz}, 6 \mathrm{H})$, $5.17(\mathrm{~m}, 1 \mathrm{H}), 5.87(\mathrm{~d}, J=0.9 \mathrm{~Hz}, 1 \mathrm{H}), 6.32(\mathrm{~d}, J=0.9 \mathrm{~Hz}, 1 \mathrm{H})$, 7.25 7.45 (m, 5H); ${ }^{13} \mathrm{C}$ NMR $\left(75 \mathrm{MHz}, \mathrm{CDCl}_{3}\right) \delta 21.7,68.5,126.1$, 128.0, 128.2, 136.8, 141.8, 166.3; IR (film): 2981, 1714, 1196, $1091 \mathrm{~cm}^{-1}$; EI-MS $(\mathrm{m} / z$, relative intensity): $190\left(\mathrm{M}^{+}, 35\right), 148$ (55), 132 (23), 103 (100), 77 (35), 43 (52); HRMS calcd for $\mathrm{C}_{12} \mathrm{H}_{14} \mathrm{O}_{2}$ 190.0994, found 190.0990 .

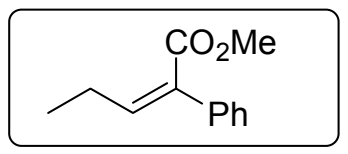

\section{(Z)-Methyl 2-phenylpent-2-enoate (5b)}

Colorless oil. ${ }^{1} \mathrm{H}$ NMR $\left(300 \mathrm{MHz}, \mathrm{CDCl}_{3}\right) \delta 1.11(\mathrm{t}, J=7.5 \mathrm{~Hz}, 3 \mathrm{H})$, $2.45(\mathrm{~m}, 2 \mathrm{H}), 3.80(\mathrm{~s}, 3 \mathrm{H}), 6.18(\mathrm{t}, J=7.5 \mathrm{~Hz}, 1 \mathrm{H}), 7.26 \sim 7.32(\mathrm{~m}, 5 \mathrm{H})$;

${ }^{13} \mathrm{C}$ NMR $\left(75 \mathrm{MHz}, \mathrm{CDCl}_{3}\right) \delta 13.8,23.6,51.7,127.2,127.5,128.2,133.9,137.9,142.1,168.6$; IR (film): 2967, 1721, $1202 \mathrm{~cm}^{-1}$; EI-MS ( $\mathrm{m} / z$, relative intensity): $190\left(\mathrm{M}^{+}, 100\right), 158(65)$, 131 (78), 115 (60), 91 (48), 77 (20); HRMS calcd for $\mathrm{C}_{12} \mathrm{H}_{14} \mathrm{O}_{2}$ 190.0994, found 190.0999.

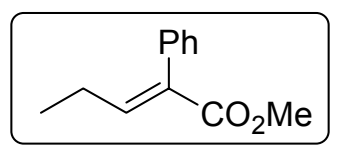

\section{(E)-Methyl 2-phenylpent-2-enoate (5b)}

Colorless oil. ${ }^{1} \mathrm{H}$ NMR $\left(300 \mathrm{MHz}, \mathrm{CDCl}_{3}\right) \delta 1.01(\mathrm{t}, J=7.5 \mathrm{~Hz}, 3 \mathrm{H})$, $2.08(\mathrm{~m}, 2 \mathrm{H}), 3.72(\mathrm{~s}, 3 \mathrm{H}), 7.07(\mathrm{t}, J=7.5 \mathrm{~Hz}, 1 \mathrm{H}), 7.16 \sim 7.18(\mathrm{~m}, 2 \mathrm{H})$, 7.31 7.39 (m, 3H); ${ }^{13} \mathrm{C} \mathrm{NMR}\left(75 \mathrm{MHz}, \mathrm{CDCl}_{3}\right) \delta 13.2,22.8,51.9,127.3,127.9,129.6,133.1$, 135.3, 146.7, 167.7; IR (film): 2967, 1716, $1241 \mathrm{~cm}^{-1}$; EI-MS ( $/ z / z$, relative intensity): 190 $\left(\mathrm{M}^{+}, 100\right), 158$ (65), 131 (79), 115 (60), 91 (49), 77 (20); HRMS calcd for $\mathrm{C}_{12} \mathrm{H}_{14} \mathrm{O}_{2}$ 190.0994, found 190.0992 .

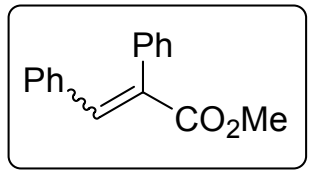

Methyl 2,3-diphenylacrylate (5c) ${ }^{10}$

White solid. $Z: E$ mixture of isomers $1: 1.5 . E$ isomer: ${ }^{1} \mathrm{H}$ NMR (300 $\left.\mathrm{MHz} \mathrm{CDCl}_{3}\right) \delta 3.79(\mathrm{~s}, 3 \mathrm{H}), 7.03 \sim 7.48(\mathrm{~m}, 10 \mathrm{H}), 7.86(\mathrm{~s}, 1 \mathrm{H})$; mixture 
of isomers: ${ }^{13} \mathrm{C}$ NMR $\left(75 \mathrm{MHz}, \mathrm{CDCl}_{3}\right) \delta 52.2,52.3,126.4,127.8,128.1,128.2,128.3,128.5$, $128.6,128.7,129.0,129.7,130.5,131.5,132.4,134.5,134.8,135.6,135.8,136.8,140.5$, 168.3, 170.0; IR (film): 3025, 2949, 1711, 1251, $1168 \mathrm{~cm}^{-1}$; EI-MS ( $\mathrm{m} / z$, relative intensity): $238\left(\mathrm{M}^{+}, 89\right), 207$ (8), 179 (78), 121 (100), 103 (14), 77 (21).

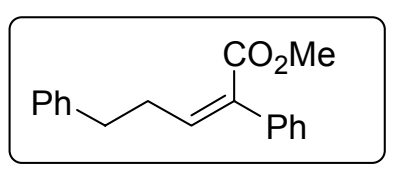

(Z)-Methyl 2,5-diphenylpent-2-enoate $(\boldsymbol{Z}-5 \mathrm{~d})$

Colorless oil. ${ }^{1} \mathrm{H}$ NMR $\left(300 \mathrm{MHz}, \mathrm{CDCl}_{3}\right) \delta 2.75 \sim 2.85(\mathrm{~m}, 4 \mathrm{H})$, $3.77(\mathrm{~s}, 3 \mathrm{H}), 6.21(\mathrm{t}, J=6.9 \mathrm{~Hz}, 1 \mathrm{H}), 7.18 \sim 7.32(\mathrm{~m}, 10 \mathrm{H}) ;{ }^{13} \mathrm{C} \mathrm{NMR}$ $\left(75 \mathrm{MHz}, \mathrm{CDCl}_{3}\right) \delta 31.8,35.4,51.7,126.0,127.3,127.6,128.2$, 128.4, 128.5 134.9, 137.9, 139.6, 141.1, 168.4; IR (film): 2920, 1720, $1204 \mathrm{~cm}^{-1}$; EI-MS (m/z, relative intensity): $266\left(\mathrm{M}^{+}, 22\right), 235$ (6), 190 (6), 115 (54), 91 (100); Anal. calcd for $\mathrm{C}_{18} \mathrm{H}_{18} \mathrm{O}_{2}$ : C, 81.17; H, 6.81. Found C, 81.18; H 6.92.

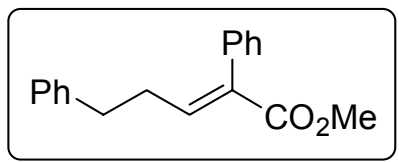

(E)-Methyl 2,5-diphenylpent-2-enoate $(\boldsymbol{E}-5 \mathrm{~d})$

Colorless oil. ${ }^{1} \mathrm{H}$ NMR $\left(300 \mathrm{MHz}, \mathrm{CDCl}_{3}\right) \delta 2.35 \sim 2.42(\mathrm{~m}, 2 \mathrm{H})$, $2.72(\mathrm{t}, J=7.5 \mathrm{~Hz}, 2 \mathrm{H}), 3.71(\mathrm{~s}, 3 \mathrm{H}), 7.03 \sim 7.35(\mathrm{~m}, 11 \mathrm{H}) ;{ }^{13} \mathrm{C}$ NMR $\left(75 \mathrm{MHz}, \mathrm{CDCl}_{3}\right) \delta 31.2,34.9,52.0,126.1,127.4,127.9$, 128.3, 128.4, 129.5, 134.3, 135.1, 140.8, 144.0, 167.6; IR (film): 2949, 1715, $1252 \mathrm{~cm}^{-1}$; EI-MS ( $\mathrm{m} / \mathrm{z}$, relative intensity): $266\left(\mathrm{M}^{+}, 41\right), 235$ (8), 175 (12), 115 (90), 91 (100), 77 (9). Anal. calcd for $\mathrm{C}_{18} \mathrm{H}_{18} \mathrm{O}_{2}$ : C, 81.17; H, 6.81. Found C, 81.13; H 6.91.

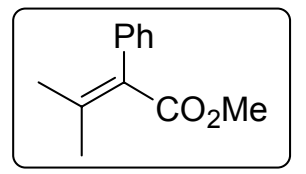

\section{Methyl 3-methyl-2-phenylbut-2-enoate (5e) ${ }^{11}$}

Colorless oil. ${ }^{1} \mathrm{H}$ NMR $\left(300 \mathrm{MHz}, \mathrm{CDCl}_{3}\right) \delta 1.71(\mathrm{~s}, 3 \mathrm{H}), 2.16(\mathrm{~s}, 3 \mathrm{H})$, $3.69(\mathrm{~s}, 3 \mathrm{H}), 7.18 \sim 7.20(\mathrm{~m}, 2 \mathrm{H}), 7.28 \sim 7.39(\mathrm{~m}, 3 \mathrm{H}) ;{ }^{13} \mathrm{C} \mathrm{NMR}(75 \mathrm{MHz}$, $\left.\mathrm{CDCl}_{3}\right) \delta 22.6,23.3,51.6,126.9,128.1,129.4,129.7,138,1$ 145.7, 168.9; IR (film): 2949, 1714, $1220 \mathrm{~cm}^{-1}$; EI-MS ( $\mathrm{m} / z$, relative intensity): $190\left(\mathrm{M}^{+}, 100\right), 158(57)$, 131 (79), 115 (57), 73 (84).

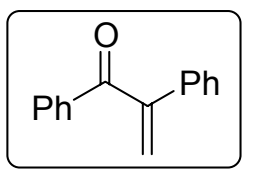

\section{1,2-Diphenylprop-2-en-1-one (5f) ${ }^{12}$}

White solid. ${ }^{1} \mathrm{H}$ NMR $\left(300 \mathrm{MHz}, \mathrm{CDCl}_{3}\right) \delta 5.64(\mathrm{~s}, 1 \mathrm{H}), 6.07(\mathrm{~s}, 1 \mathrm{H})$, 7.33 7.57 (m, 8H), 7.90 7.93 (m, 2H); $\left.{ }^{13} \mathrm{C} \mathrm{NMR} \mathrm{(75} \mathrm{MHz,} \mathrm{CDCl}_{3}\right) \delta 120.9$, $127.0,128.3,128.4,128.6,129.9,133.0,136.9,137.0,148.2$, 197.5; IR (film): 3059, 1665, $1214 \mathrm{~cm}^{-1}$; EI-MS ( $\mathrm{m} / \mathrm{z}$, relative intensity): $208\left(\mathrm{M}^{+}, 38\right), 132$ (4), 105 (100), 77 (59).

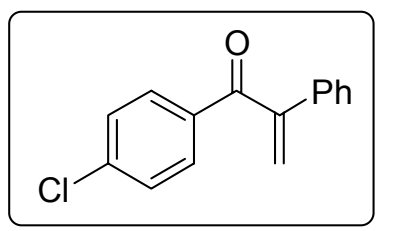

\section{1-(4-Chlorophenyl)-2-phenylprop-2-en-1-one (5g)}

Pale yellow oil. ${ }^{1} \mathrm{H}$ NMR $\left(300 \mathrm{MHz}, \mathrm{CDCl}_{3}\right) \delta 5.64(\mathrm{~s}, 1 \mathrm{H}), 6.06(\mathrm{~s}$, $1 \mathrm{H}), 7.32 \sim 7.42(\mathrm{~m}, 7 \mathrm{H}), 7.82 \sim 7.87(\mathrm{~m}, 2 \mathrm{H}) ;{ }^{13} \mathrm{C} \mathrm{NMR}(75 \mathrm{MHz}$, $\left.\mathrm{CDCl}_{3}\right) \delta 121.1,126.9,128.5,128.6,128.7,131.3,135.3,136.7$, 139.5, 147.9, 196.2; IR (film): 3059, 1665, 1586, $1212 \mathrm{~cm}^{-1}$; EI-MS ( $m / z$, relative intensity): $242\left(\mathrm{M}^{+}, 42\right), 207$ (20), 139 (100), 111 (26), 103 (25); HRMS calcd for $\mathrm{C}_{15} \mathrm{H}_{11} \mathrm{OCl} 242.0498$, found 242.0492. 


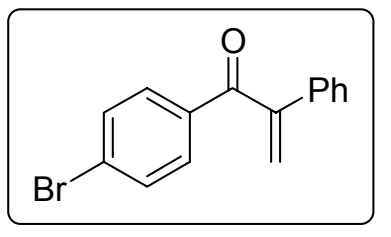

\section{1-(4-Bromophenyl)-2-phenylprop-2-en-1-one (5h)}

Pale yellow oil. ${ }^{1} \mathrm{H}$ NMR $\left(300 \mathrm{MHz}, \mathrm{CDCl}_{3}\right) \delta 5.65(\mathrm{~s}, 1 \mathrm{H}), 6.07(\mathrm{~s}$, $1 \mathrm{H}), 7.32 \sim 7.42(\mathrm{~m}, 5 \mathrm{H}), 7.54 \sim 7.58(\mathrm{~m}, 2 \mathrm{H}), 7.74 \sim 7.79(\mathrm{~m}, 2 \mathrm{H}) ;{ }^{13} \mathrm{C}$ NMR $\left(75 \mathrm{MHz}, \mathrm{CDCl}_{3}\right) \delta 121.1,126.9,128.2,128.5,128.6,131.4$, 131.7, 135.7, 136.6, 147.9, 196.3; IR (film): 3057, 1665, 1583, 1210 $\mathrm{cm}^{-1}$; EI-MS ( $\mathrm{m} / z$, relative intensity): $286\left(\mathrm{M}^{+}, 25\right), 207$ (57), 183 (100), 155 (27), 103 (42); HRMS calcd for $\mathrm{C}_{15} \mathrm{H}_{11} \mathrm{OBr} 285.9993$, found 285.9987.

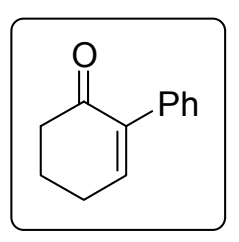

\section{2-Phenylcyclohex-2-enone (5i) ${ }^{13}$}

Pale yellow solid. ${ }^{1} \mathrm{H}$ NMR $\left(300 \mathrm{MHz}, \mathrm{CDCl}_{3}\right) \delta 2.05 \sim 2.14(\mathrm{~m}, 2 \mathrm{H})$, 2.50 2.61 (m, 4H), $7.03(\mathrm{t}, J=4.2 \mathrm{~Hz}, 1 \mathrm{H}), 7.25 \sim 7.37(\mathrm{~m}, 5 \mathrm{H}) ;{ }^{13} \mathrm{C}$ NMR $(75$ $\left.\mathrm{MHz}, \mathrm{CDCl}_{3}\right) \delta 22.8,26.5,39.0,127.5,127.9,128.6,136.5,140.3,148.0$, 197.9; IR (film): 2941, 1680, $1154 \mathrm{~cm}^{-1}$; EI-MS ( $\mathrm{m} / z$, relative intensity): 172 $\left(\mathrm{M}^{+}, 100\right), 144$ (74), $116(77), 89(10), 77$ (13).

\section{$\operatorname{Pd}\left(\mathrm{PPh}_{3}\right)_{4}$-Catalyzed Reaction of Diazo Compond 1 with Iodobenzene}

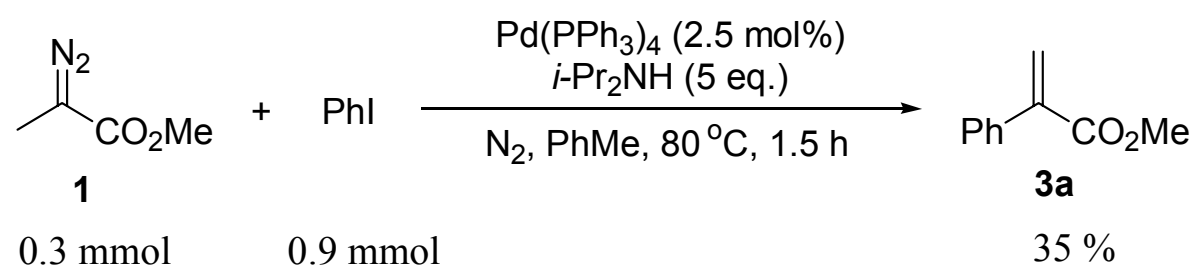

\section{References}

(1) Aller, E.; Brown, D. S.; Cox, G. G.; Miller, D. J.; Moody, C. J. J. Org. Chem. 1995, 60, 4449.

(2) Taber, D. F.; Ruckle Jr., R. E.; Hennessy, M. J. J. Org. Chem. 1986, 51, 4077.

(3) Selva, M.; Tundo, P. J. Org. Chem. 2006, 71, 1464.

(4) Jefford, C. W.; Bernardinelli, G.; Wang, Y.; Spellmeyer, D. C.; Buda, A.; Houk, K. N. J. Am. Chem. Soc. 1992, 114, 1157.

(5) Levin, J. I. Tetrahedron Lett. 1993, 34, 6211.

(6) Silveira, P. B. Monteiro, A. L. J. Mol. Catal. A: Chemical 2006, 247, 1.

(7) Chang, M. Y.; Chen, S. T.; Chang, N. C. Tetrahedron 2002, 58, 3623.

(8) Zhang, C.; Ito, H.; Maeda, Y.; Shirai, N.; Ikeda, S.; Sato, Y. J. Org. Chem. 1999, 64, 581.

(9) Nishimura, K.; Ono, M.; Nagaoka, Y.; Tomioka, K. Angew. Chem. Int. Ed. 2001, 40, 440.

(10) Reger, D. L.; Mintz, E.; Lebioda, L. J. Am. Chem. Soc. 1986, 108, 1940.

(11) Jacobi, P. A.; Brielmann, H. L.; Cann, R. O. J. Org. Chem. 1994, 59, 5305.

(12) Hon, Y. S.; Hsu, T. R.; Chen, C. Y.; Lin, Y. H.; Chang, F. J.; Hsieh, C. H.; Szu, P. H. Tetrahedron 2003, 59, 1509.

(13) Felpin, F. -X. J. Org. Chem. 2005, 70, 8575. 